\title{
STUDY OF ZMP MEASUREMENT FOR BIPEDROBOT USING FSR SENSOR
}

\author{
Bong Gyou Shim, *Eung Hyuk Lee, **Hong Ki Min, Seung Hong Hong \\ Department of Electronic Engineering, Inha University \\ * Department of Electronic Engineering, Korea Polytechnic University \\ ** Department of Information \& Telecom Engineering, University of Incheon \\ E-mail : bipedrobot@empal.com, *ehlee@kpu.ac.kr,**mkmin@incheon.ac.kr, \\ shhong@inha.ac.kr
}

\begin{abstract}
This paper discuss a method measuring ZMP for biped robot. We obtained feet-bottom pressure distribution using FSR sensors attaching feet-bottom. We calculate value of ZMP coordinates that is the center of gravity for biped robot using their pressure distribution. Value of Biped Robot ZMP coordinates always exists in feet-bottom. When walking limbs of biped robot are controlled, inverse kinematics use method supposed by author. We introduce it in this paper.
\end{abstract}

Keywords: Biped Robot, FSR Sensor, ZMP, feet-bottom

\section{INTRODUCTION}

To improve life quality, human request robot that can alternate one's work. This robot is calling humanoid. Robot Technology goal is humanoid. We may try humanoid robot being. Recently, study of humanoid robot is proceeding in many place. Generation of car model robot driven by wheels sooner or later replace with wakling robot generation. And, we might oppose that we may replace human effort as role of home robot as well as industry robot. Walking is a essential basal condition so that anthropomorphic robot adapts to human environment.

In this study, author formerly makes a small-sized humanoid oneself. This humanoid names DORRAE. Humanoid DORRAE is korean that means pulley. Pulley symbolizes balance and control of force. From a robot kinematical point of view, DORRAE is robot which was made farther nearly human structure. Existing DORRAE gait formula is method realized via sequential pattern tracing using walking pattern reference. But, the center of gravity caused by robot weight, that is, Zero Moment Point(ZMP) must be measured and controlled in realtime operation so that robot can overcome to tumble due to unexpected noise. therefore, measuring ZMP is preceded.

In this paper, when we control humanoid robot walking, the upper limbs is fixed and the nether limbs is controlled using inverse-kinematics method proposed by author. In this condition, for measuring and maintaining ZMP, we use to attach four FSR sensors a foot in exiting study but three FSR sensors a foot in this paper. This paper experiment shows measurement ability to attach three FSR sensors per foot.

\section{ABOUT DORRAE ROBOT}

DORRAE appearance is showed by figure 1 .

Special feature of DORRAE is 1) autonomous locomotion 2) remote robot control(using Bluetooth) 3) realization of varying posture made by twenty joint.

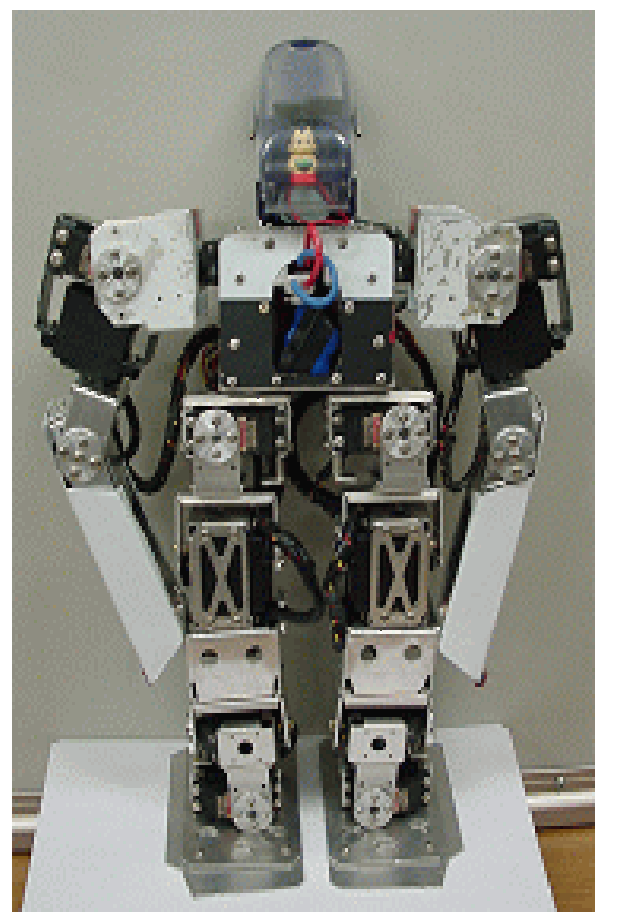

Figure 1. Biped robot made for experiment

DORRAE has twenty degree of freedom(DOF) which consist of each two unit that arm has four DOF and leg has six DOF. Height is $350 \mathrm{~mm}$ and weight is $2.5 \mathrm{~kg}$. DORRAE joint organization is like figure 2 . 
Main processor of DORRAE is ATmega128 which is RISC 8bit prosessor AVR. Therefore, we can remote control using Bluetooth.

If robot tumbled, Robot can pick oneself up as it trace sequential pattern reference.

In the future, when robot is tumbled, it will add function picking oneself up via balance and gyro sensor.

\begin{tabular}{|c|c|c|c|}
\hline \multicolumn{3}{|c|}{ Joint } & quantity \\
\hline \multirow{3}{*}{\multicolumn{2}{|c|}{ head }} & roll & 0 \\
\hline & & pitch & 0 \\
\hline & & yaw & 0 \\
\hline \multirow{8}{*}{ arm } & \multirow{3}{*}{ shoulder } & roll & 2 \\
\hline & & pitch & 2 \\
\hline & & yaw & 2 \\
\hline & \multirow{2}{*}{ elbow } & roll & 0 \\
\hline & & pitch & 2 \\
\hline & \multirow{2}{*}{ wrist } & roll & 0 \\
\hline & & pitch & 0 \\
\hline & hand & pitch & 0 \\
\hline \multirow{3}{*}{\multicolumn{2}{|c|}{ waist }} & roll & 0 \\
\hline & & pitch & 0 \\
\hline & & yaw & 0 \\
\hline \multirow{6}{*}{ leg } & \multirow{3}{*}{ hip } & roll & 2 \\
\hline & & pitch & 2 \\
\hline & & yaw & 2 \\
\hline & knee & pitch & 2 \\
\hline & \multirow{2}{*}{ ankle } & roll & 2 \\
\hline & & pitch & 2 \\
\hline
\end{tabular}

figure 2. DORRAE joint formation

\section{JOINT CONTROL}

DORRAE is modeling like figure 2.

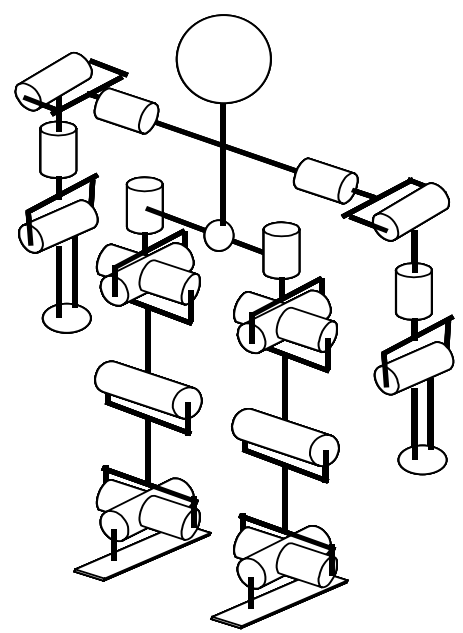

Figure 2. Biped robot(DORRAE) model
In order to control robot, kinematics modeling must be preceded. Generally, biped robot is been modeling as kinematical analysis divisor like DenavitHartenberg notation(D-H notation) applying to manipulator(robot arm) which often use the progress of industrial work. But it is difficult to realize lowcost microprocessor because this exiting methods have long operation time. By the way, Walking human leg is working space and motion is more limitary than manipulator. Thus, modeling method which can reduce operation time was issued by author. [4]

Simply to discuss this, Walking demanded motion of leg regard as three motion. The three motion is to regulate leg length using knee, to move foot to front or back and to move foot to left or right. Thus, we obtain angle of each joint through degree of the three motion.

\subsection{Re-modeling about leg length}

Prismatic joint structure regard as knee joint of robot like figure 3 , and then it analyzes.

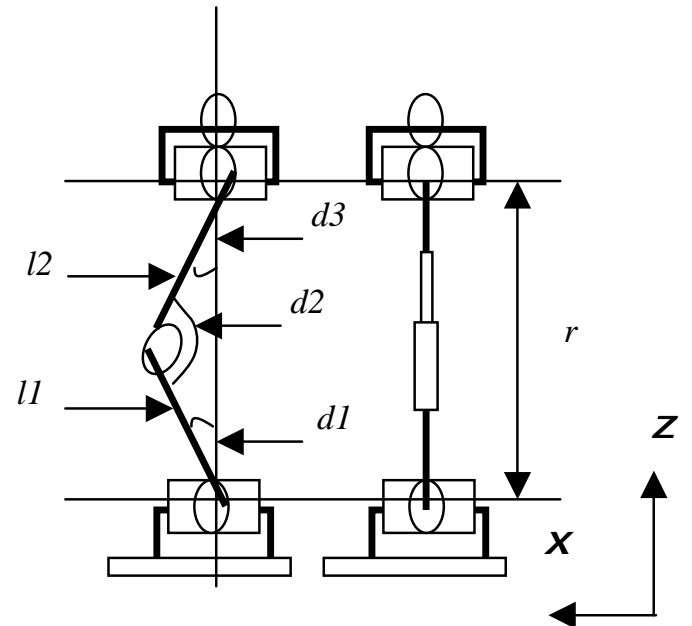

Figure 3. Re-modeling to regulate leg length using knee

3.2 Re-modeling to move foot to front or back

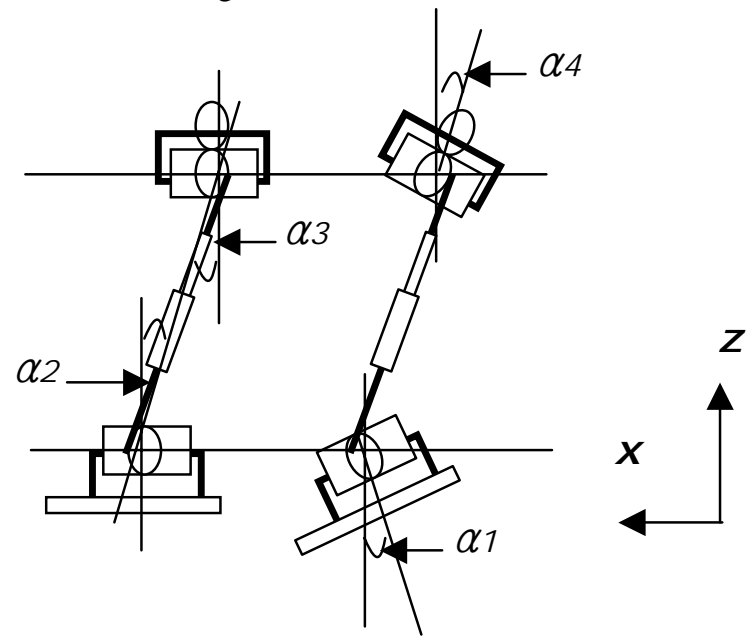

Figure 4. Re-modeling to move foot to front or back 
Like as figure 4 , four variables which is $\alpha 1, \alpha 2$, $\alpha 3, \quad \alpha 4$ assign in two joint took part in motion to move foot to front or back. $\alpha 2, \alpha 3$ move to same angle and $\alpha 1, \alpha 4$ indicate direction angle of the upper and lower each joint. By $\alpha 2(=\alpha 3)$, The value of $\mathrm{x}$ coordinates from center to foot is obtained.

\subsection{Re-modeling to move foot to left or right}

Like as figure 5 , four variables which is $\delta 1, \delta 2$, $\delta 3, \delta 4$ assign in two joint took part in motion to move foot to front or back. $\delta 2, \delta 3$ move to same angle and $\alpha 1, \alpha 4$ indicate direction angle of the upper and lower each joint. By $\delta 2(=\delta 3)$, The value of y coordinates from center to foot is obtained.

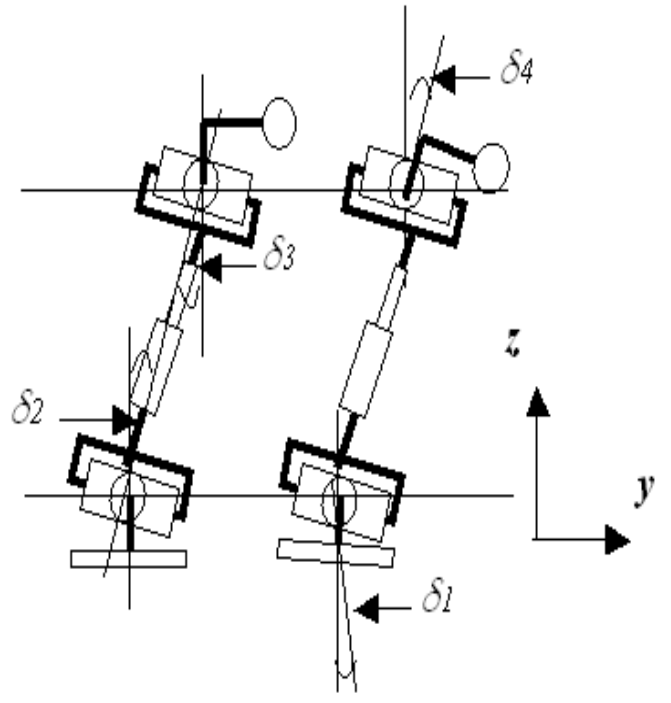

Figure 5. Re-modeling to move foot to left or right

\section{ZMP MEASUREMENT}

We obtained feet-bottom pressure distribution using FSR sensors attaching feet-bottom. We calculate value of ZMP(Zero Moment Point) coordinates that is the center of gravity for biped robot using their pressure distribution.

Formerly ZMP Measurement is used to attach feetbottom to four FSR sensors like Figure 3. In this paper, it is used for ZMP Measurement to attach feetbottom to three FSR sensors like Figure 4. We made a experiment foot like Figure 9.(b) and equation to calculate pressure about each coordinates. We lay a counterweight in each coordinates and then measured all FSR value. We contrasted pressure value based on equation with measuring pressure value. Using experiment result we made equation to calculate ZMP coordinates value about each FSR sensor value. This experiment show that equation to calculate ZMP coordinates value proposed by author is adequate.

4.1 The existing method of ZMP measurement In order to obtain precise ZMP, we install accelerometer in the center of all link gravity and then calculation ZMP using value measured these. But, this method is difficult to realize system. more simplicity and efficient method is to measure ZMP using FSR(Force Sensing Resistors). Method measuring ZMP using FSR seek the center of gravity. Using FSR, we easily trace movement of ZMP. Like as equation (1), we can obtain the coordinate values of ZMP.

$$
\begin{aligned}
& x_{Z M P}=\frac{\sum_{i=1}^{4} f_{i} x_{i}}{\sum_{i=1}^{4} f_{i}} \\
& y_{Z M P}=\frac{\sum_{i=1}^{4} f_{i J i}}{\sum_{i=1}^{4} f_{i}}
\end{aligned}
$$

When we calculate the coordinate values of ZMP, absolute magnitude of force measured via FSR isn't significant. It is because relative value is decided.
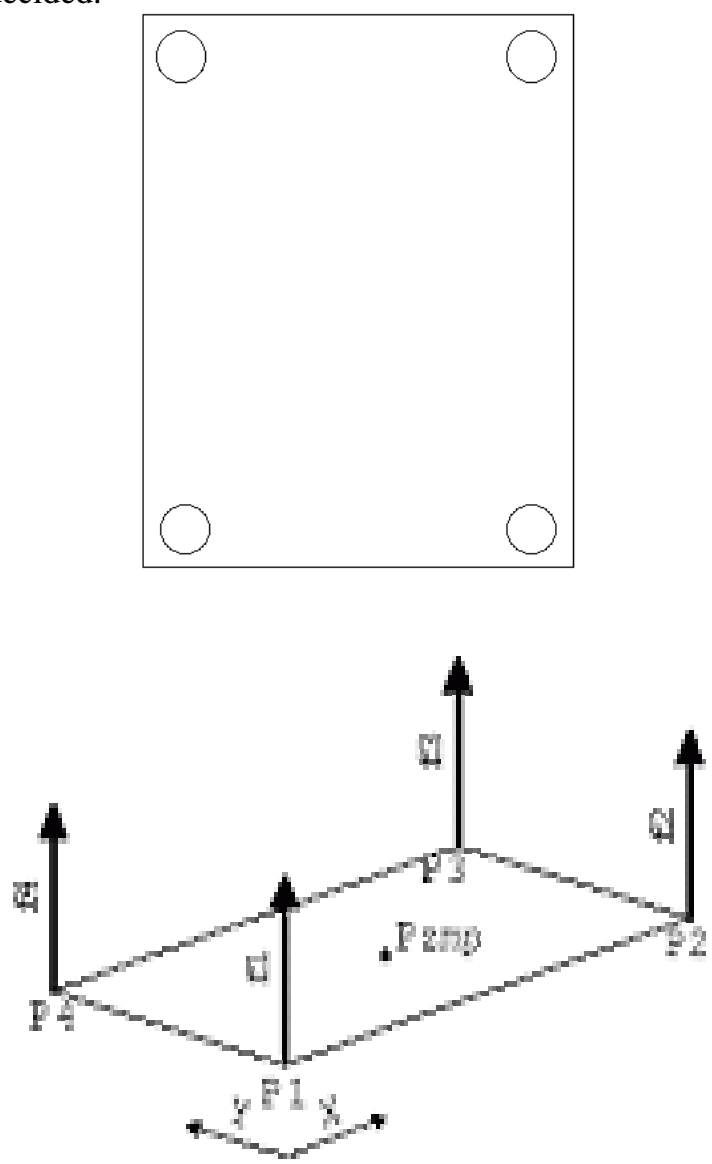

Figure 6. Attached four FSR sensors a foot

In figure $6, a, b, c, d$ are measured values via $\mathrm{p} 1$, p2, p3, p4. When na, nb, nc, nd ( $n$ is optional integer), we anticipate to calculate same ZMP constantly. Hence this has two meaning.

First, If force values measured via each FSR is very 
small, ZMP seriously is changed by measured error. Second, with make foot-bottom precisely it must have structure that force distribution uniform. If it isn't so, measured ZMP values are imprecise.

4.2 The improved method of ZMP measurement When measured FSR values are $\mathrm{f} 1>\mathrm{fmin}, \mathrm{f} 2>\mathrm{fmin}$, $\mathrm{f} 3>\mathrm{fmin}$, f4 $>$ fmin, value of optional one - for instance, $\mathrm{f} 1$ - can alternatives value used by equation (2). Thus, to use three FSR that isn't four FSR can reduce the number of FSR. It is proved by experiments executed in this paper.

$\mathrm{f} 1=\mathrm{f} 2 \times \mathrm{f} 4 / \mathrm{f} 3$

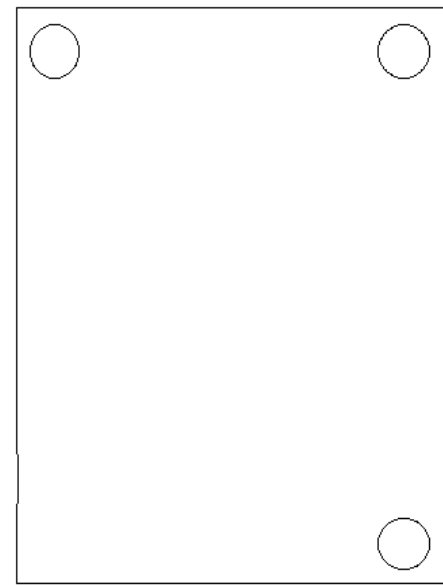

Figure 7. Attached Three FSR sensors a foot

4.3 Design of biped robot foot-bottom for ZMP measuring

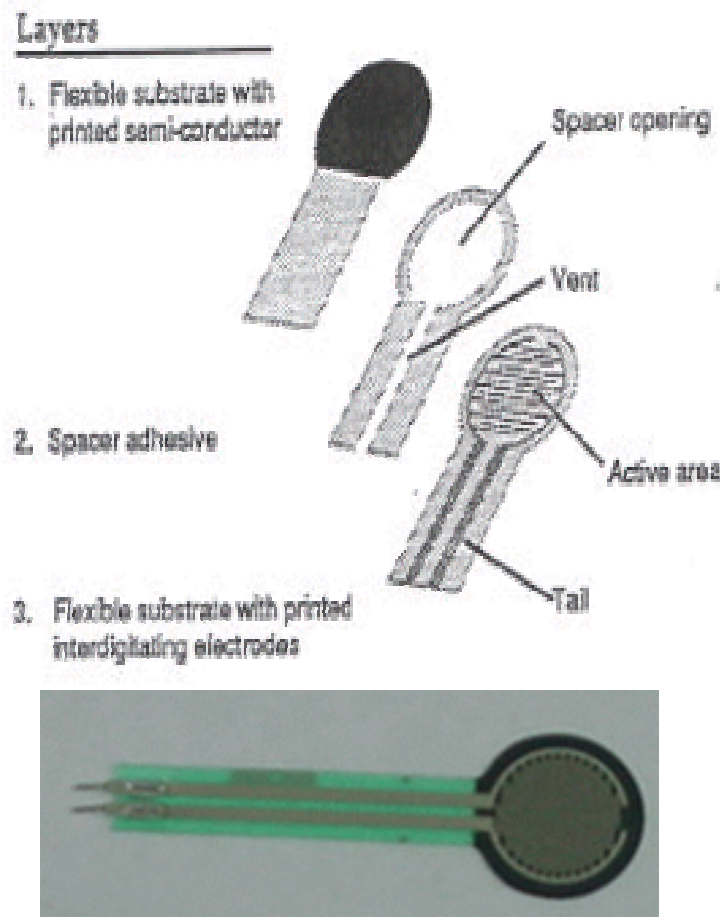

Figure 8. FSR sensors attaching in biped robot footbottom
Shown figure 8, force Sensing Resistors(FSR) are a polymer thick film (PTF) device which exhibits a decrease in resistance with an increase in the force applied to the active surface. Its force sensitivity is optimized for use in human touch control of electronic devices. FSRs are not a load cell or strain gauge, though they have similar properties. FSRs are not suitable for precision measurements.

When we calculate the coordinate values of ZMP, absolute magnitude of force measured via FSR isn't significant. It is because relative value is decided.

Like as figure 9.(a), length(axis of $\mathrm{x}$ ) is $120 \mathrm{~mm}$, width(axis of y) is $80 \mathrm{~mm}$, and four corner which support upper body of foot-bottom are a regular square that length of a side is $25 \mathrm{~mm}$. Both surface attaching FSR select material to be able to press on it softly then to absorb shock. figure 9.(b) is the picture of feet-bottom made by author.

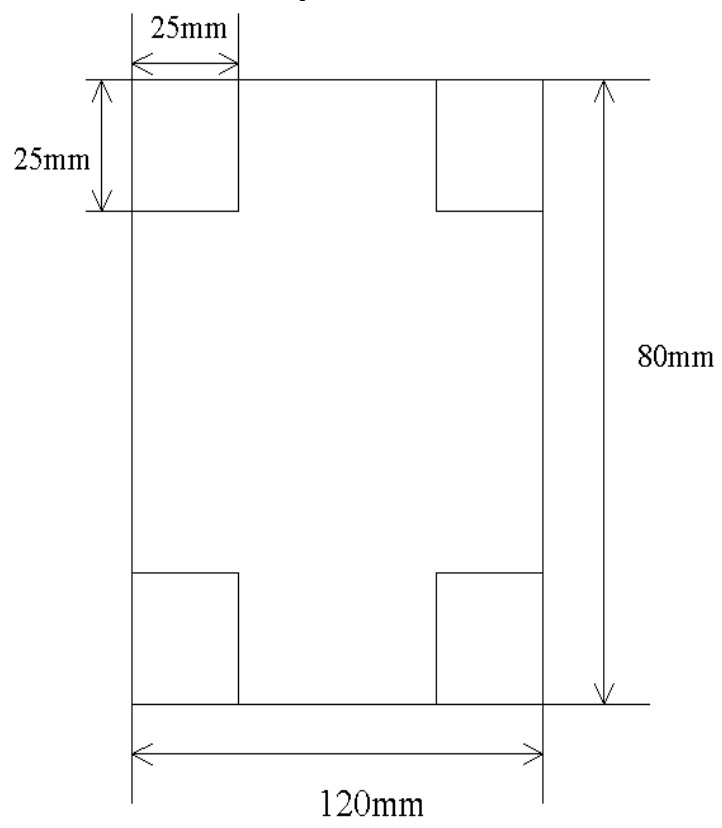

(a) Standard of foot-bottom

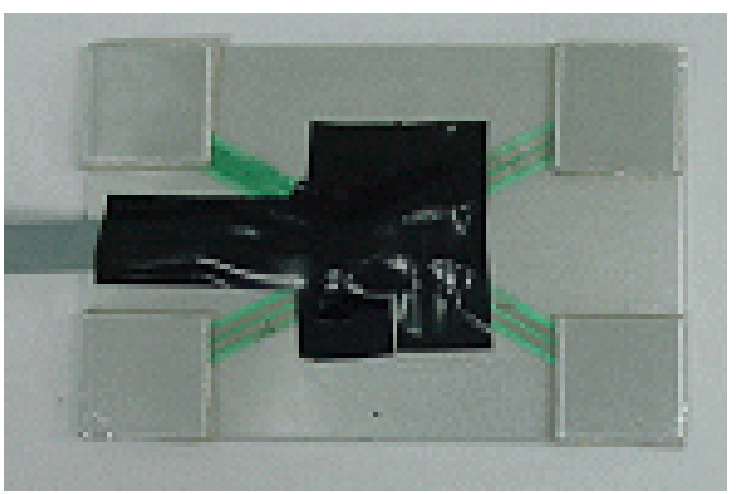

(b) Picture of foot-bottom

Figure 9. Foot-bottom attached Four FSR Sensors 
4.4 ZMP measuring experiment

The value of coordinates for experiment is that axis of $\mathrm{x}$ is value from $+40 \mathrm{~mm}$ to $-40 \mathrm{~mm}$ maintaining $10 \mathrm{~mm}$ gap and axis of $\mathrm{y}$ is value from $+20 \mathrm{~mm}$ to $20 \mathrm{~mm}$ maintaining $5 \mathrm{~mm}$ gap. This is the appointed value of coordinates(o).

When it is inflicted that weight of $3 \mathrm{~kg}$ on surface that the appointed value of coordinates is the center of circle and a diameter is $10 \mathrm{~mm}$, pressure value of $\mathrm{f} 1, \mathrm{f} 2, \mathrm{f} 3, \mathrm{f} 4$ is measured. To assign $\mathrm{f} 1, \mathrm{f} 2, \mathrm{f} 3, \mathrm{f} 4$ to equation (1), the measured value of coordinates $(+)$ can be obtained. To subtract the appointed value of coordinates(o) from the measured value of coordinates( $(+)$, an error be obtained. Figure 10 shows the appointed value of coordinates(o) and the measured value of coordinates $(+)$.

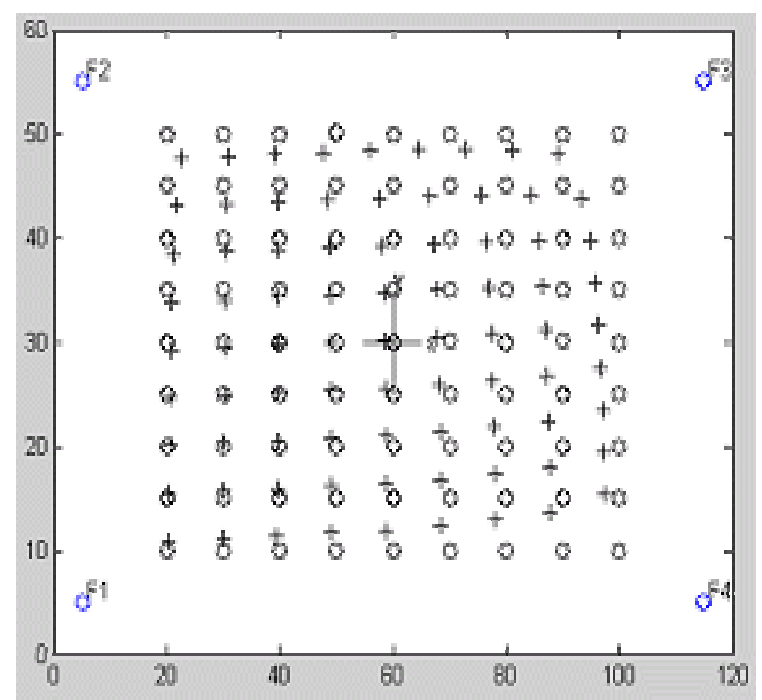

Figure 10. The measured value of coordinates(+) using four FSRs about he appointed value of coordinates(o)

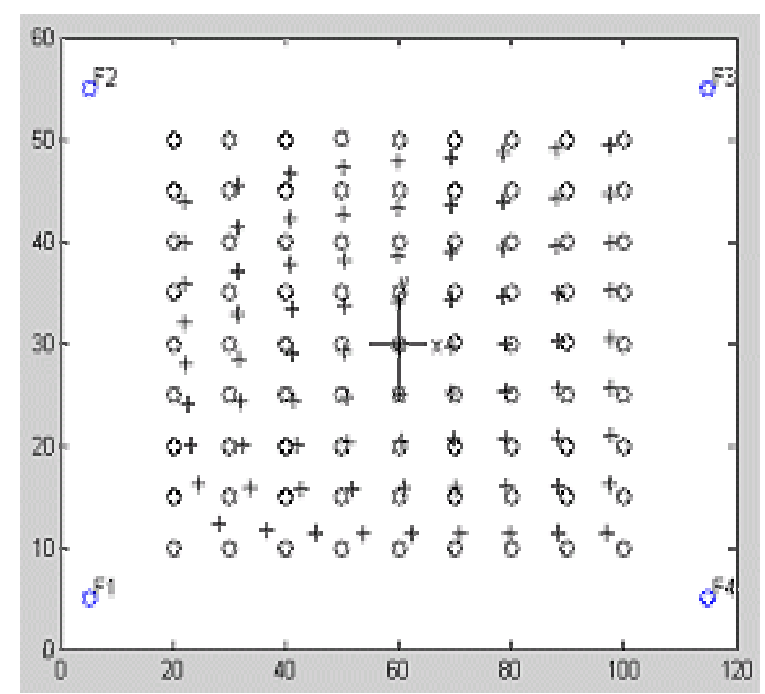

Figure 11. The appointed value of coordinates(o) and the measured value of coordinates $(+)$ using three FSRs that F1 be replaced by equation (2).
In this way, the measured value of coordinates $(+)$ is obtained to replace one by one input value of FSR as equation (2). although we remove one among $\mathrm{F} 1$, F2, F3, F4, the result doesn't very differ from using four sensor and other three sensor. Figure 11 shows the appointed value of coordinates(o) and the measured value of coordinates(+) using three FSR that F1 be replaced by equation (2).

\subsection{The result of experiment}

To subtract the appointed value of coordinates(o) from the measured value of coordinates $(+)$ in section 4.4 , an error be obtained. figure 12 shows average errors.

\begin{tabular}{|c|c|c|}
\hline \multirow{2}{*}{ used FSRs } & \multicolumn{2}{|c|}{ average error(mm) } \\
\cline { 2 - 3 } & coordinates of $x$ & coordinates of $y$ \\
\hline F1, F2, F3, F4 & -1.97 & -0.67 \\
\hline F2, F3, F4 & -3.04 & -0.79 \\
\hline F1, F3, F4 & -1.83 & 0.01 \\
\hline F1, F2, F4 & -2.03 & -0.50 \\
\hline F1, F2, F3 & -3.71 & -0.65 \\
\hline
\end{tabular}

Figure 12. Average errors with used FSRs

As a result of this experiment, when we measure the center of robot gravity, although we use optional three FSRs among four FSRs, these errors don't cause problem which make tumble robot. Thus, this experiment shows that to measure ZMP using this method isn't influence.

\section{CONCLUSION}

Processors of DORRAE are ATmega128 which is RISC 8bit prosessor AVR. Prodessors are the number of two. one is main processor which control motion pattern. another is slave processor which control the 32 of RC-servo simultaneously. unsynchronous serial communication is used between main processor and slave processor.

In the result, to attach three FSRs which is four FSRs in foot-bottom , we can measure ZMP. Thus, for the first time, humanoid can flexibly adjust against the ground condition and the force which is caused to outer disturbance.

Hence, compensation algorithm in static and dynamic status should study. 


\section{REFERENCES}

[1] John J.Craig, "Introduction To Robotics", Addison-Wesley Publishing Company, 1989.

[2] S.Hashimoto S Narita, T.Kobayashi, A. Takanishi, J. Yamaguchi, P. Dario and $\mathrm{H}$. Takanobu :Development of a Biped Humanoid Robot "WABIAN", Video Proc. Of the 1998 ICRA, 1998.

[3] S.Kajita and K.Tani, "Experimental Study of Biped Dynamic Walking", IEEE Control Systems Magazine, Vol. 16, No. 1, pp. 13-19, 1996.

[4] Bong Gyou Shim, Hyeon Min Shim, Se Kee Kil, Eung Hyuk Lee, Seung Hong Hong, "Static Gait Control of Anthropomorphic Biped Robot Based on Kinematical Re-modeling and ZMP Control", HCI Conference, Vol. 1, No. 2, pp.245-251 , 2004

[5] M Hirose, "Development of Humanoid Robot ASIMO," Proc. Int. Confernce on Intelligent Robots and Systems, Workshop2 2001.

[6] H.Inoue et al., HRP: Humanoid Robotics Project of MITI, IEEE Humanoid 2000

[7] K. Hirai, "Current and Future Perspective of Honda Humanoid Robot", Proc. IEEE/RSJ Int. Conference on Intelligent Robots and systems, PP.500-508, 1997. 